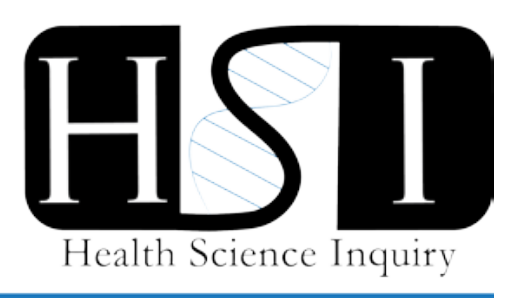

\title{
Plant-based diet as a means to prevent and treat chronic disease
}

\author{
Hosung (Joel) Kang ${ }^{1}$, Kirsten Dillon ${ }^{2}$ \\ ${ }^{1}$ Department of Health and Rehabilitation Sciences, University of Western Ontario; ${ }^{2}$ Department of Kinesiology, \\ University of Western Ontario
}

Chronic diseases, such as coronary artery disease, ischemic stroke, diabetes, and some specific types of cancer, were once thought to be prevalent in high-income countries only. However, research shows that these chronic diseases are now affecting populations worldwide [1]. Furthermore, migrants who go from a low-income country to a highincome country often have a dramatic increase in the rate of cancer and cardiovascular disease [2]. Rates of chronic disease among African Americans are similar to Caucasian Americans even though the rate of chronic disease is extremely low in traditional African societies. Not only does it illustrate the cultural environment that promote chronic disease in North America, it also indicates the primary determinants of these diseases are heavily influenced by modifiable environmental factors, such as lifestyle and diet.

Proper diet is essential to prevent chronic diseases. Although there are disagreements as to what the optimal diet is, the Adventist Health study showed that as you incrementally remove more animal products from your diet, you increasingly reduce the risk of developing diabetes [3]. To expand on this, the incidence rate of diabetes was lowest among individuals who identified themselves as vegan, which entails no animal products in their diet. However, the risk increases to those that consume only eggs and dairy (lacto-ovo vegetarian), and again heightens for those consuming fish (pesco-vegetarian). Furthermore, an even higher risk was displayed in a diet that is primarily vegetarian but does incorporate some meat occasionally (semi-vegetarian). Lastly, the typical American nonvegetarian diet, that consists of eating mostly animal based foods on a regular basis ultimately had the highest risk for developing diabetes. Furthermore, in the same study, they found that the vegetarian diet was associated with lower all cause-mortality and disease specific mortality compared with the non-vegetarian dietary pattern [4]. They also demonstrated some associations with lower mortality of the pesco-vegetarian, vegan, and lacto-ovo vegetarian diets when compared with the non-vegetarian diet. Moreover, the incidence rate of colorectal cancer was lower among vegetarian group compared with non- vegetarian group [5]. Pesco-vegetarian had the lowest incidence of colorectal cancer suggesting that fish may provide more protection than meat. This is all highlighted with Canada's newly remodeled food guidelines, removing "poultry" and replacing it with "protein"; recommending reducing and limit the intake of meat products and increase the consumption of plant-based proteins [6]. The World Health Organization (WHO) has even labelled processed meat as carcinogenic [7]. As a result, to prevent chronic diseases, it is recommended to consume more plant-based sources and limit the intake of meat and dairy products, as dairy has also been removed from the guidelines.

The importance of proper diet cannot be expressed enough to prevent the development of chronic diseases. Just like the WHO's guidelines for moderate to vigorous physical activity, argumentatively, diet needs be emphasized even more. Not only can proper diet add to longevity, it can treat and even reverse chronic diseases [8]. A recent study recruited participants with type 2 diabetes and divided them into either an experimental group or control group [8]. Both the experimental group and the control group received an isocaloric $(-500 \mathrm{kcal} /$ day $)$ diet. The experimental group received a vegetarian diet whereas the control group received a diet that consisted of foods recommended by conventional diabetic diet. A calorie restricted vegetarian diet had significantly greater improvements to patients' clinical measures than the calorie restricted conventional diabetic diet. Not only did the experimental group lose more weight with the same amount of caloric restriction, they made significant reductions in their diabetes medication, as well as significant improvements in their insulin sensitivity. Lastly, there were significant improvements in both visceral and subcutaneous fat compositions. Therefore, not only does the vegetarian diet provide greater protection from chronic diseases, it also provides improved clinical patient measures than conventional diets.

With the current demographic of our population shifting strongly towards older adults, the numerous comorbidities that develop within this age group place a huge financial burden on the current healthcare system. As a result of 
physical limitations and chronic disease, older adults will be transitioning into an assisted living home (9). That being said, it is very hard to get this population to meet the WHO guidelines for physical activity (10), which further emphasizes the importance and impact that food can have in these facilities. However, the meals provided are usually not ideal for the various types of chronic diseases. Policy makers should be concerned with what kind of food facilities are providing, as this could be a simple transition that could result in a huge impact.

\section{References}

[1] Guilbert, J. J. (2003) The world health report 2002 - reducing risks, promoting healthy life. Educ Health (Abingdon), 16 (2), 230.

[2] Willett, W. C., Koplan, J. P., Nugent, R., Dusenbury, C., Puska, P., Gaziano, T. A. (2006) Prevention of Chronic Disease by Means of Diet and Lifestye Changes. In: Jamison, D. T., Breman, J. G., Measham, A. R., Alleyne, G., Claeson, M., Evans, D. B., et al. (2006) Disease Control Priorities in Developing Countries. 2nd ed. Washington (DC), World Bank. Available from: http://www.ncbi.nlm.nih.gov/books/NBK11795/.

[3] Tonstad, S., Stewart, K., Oda, K., Batech, M., Herring, R. P., Fraser, G. E. (2013) Vegetarian diets and incidence of diabetes in the Adventist Health Study-2. Nutr Metab Cardiovasc Dis., 23 (4), 292299.

[4] Orlich, M. J., Singh, P. N., Sabaté, J., Jaceldo-Siegl, K., Fan, J., Knutsen, S., et al. (2013) Vegetarian
Dietary Patterns and Mortality in Adventist Health Study 2. JAMA Intern Med., 173 (13), 12301238.

[5] Orlich, M. J., Singh, P. N., Sabaté, J., Fan, J., Sveen, L., Bennett, H., et al. (2015) Vegetarian dietary patterns and the risk of colorectal cancers. JAMA Intern Med, 175 (5), 767776.

[6] Lancet Countdown 2017 Report: Briefing for Canadian Policymakers. Canadian Public Health Association, 2017, 1-25.

[7] Bouvard, V., Loomis, D., Guyton, K. Z., Grosse, Y., Ghissassi, F. E., Benbrahim-Tallaa, L., et al. (2015) Carcinogenicity of consumption of red and processed meat. The Lancet Oncology, 16 (16), 15991600.

[8] Kahleova, H., Matoulek, M., Malinska, H., Oliyarnik, O., Kazdova, L., Neskudla, T., et al. (2011) Vegetarian diet improves insulin resistance and oxidative stress markers more than conventional diet in subjects with Type 2 diabetes. Diabet Med., 28 (5), 549559.

[9] Leung, P-M., Ejupi, A., van Schooten, K. S., Aziz, O., Feldman, F., Mackey, D. C., et al. (2017) Association between Sedentary Behaviour and Physical, Cognitive, and Psychosocial Status among Older Adults in Assisted Living. Biomed Res Int, 2017: 9160504. [Available from: https://www.ncbi.nlm.nih.gov/pmc/articles/PMC55879 $24 /$.

[10] Directly measured physical activity of adults, 2012 and 2013. Government of Canada SC, 2015. Available from: https://www.statcan.gc.ca/pub/82625-x/2015001/article/14135-eng.htm. 


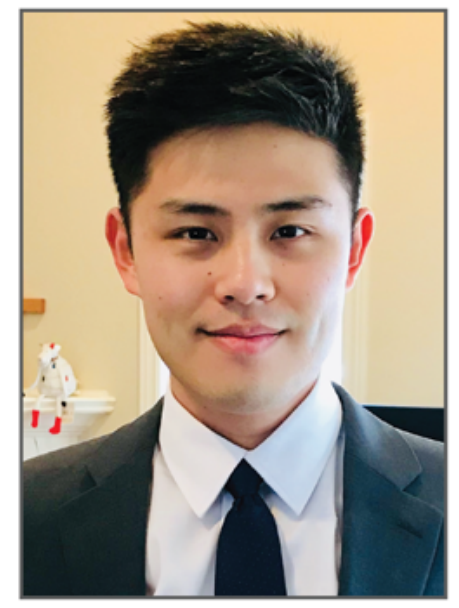

Hosung (Joel) completed his honours BSc with distinction from the department of Neuroscience at the University of Western Ontario. He subsequently began his MSc degree at the University of Western Ontario transitioning his research focus to Health and Rehabilitation Sciences. He will primarily be studying the organization of healthcare teams and try to understand the factors that contribute to high-performing teams.

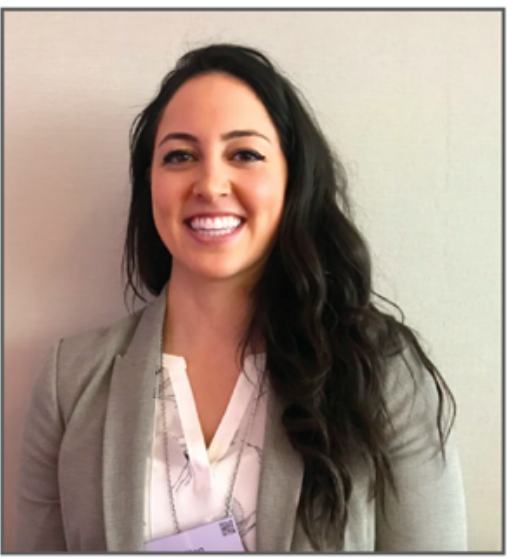

Kirsten Dillon is a Master's student at the University of Western Ontario. As a researcher in the Exercise and Health Psychology Laboratory, her primary focus is the determination of how reducing sedentary behaviour in older adults will affect cognitive decline, physical functioning, sleep and quality of life. Additionally, her research involves using exercise as a means to treat and prevent chronic disease in all populations. Kirsten completed her B.Sc. (hons.) in Kinesiology and Health Science and Psychology at York University while simultaneously captaining the women's varsity volleyball team for five years. 